psychotic versus non-psychotic depression has not been systematically studied. We therefore examined B12 and folate levels in unmedicated patients with well-defined major depression to determine the association between psychotic depression and serum levels of these vitamins.

Of 53 patients presenting to the Mood Disorders Clinic with a major depressive episode as defined by Research Diagnostic Criteria (Spitzer et al, 1977) generated from the Schedule for Affective Disorders and Schizophrenia - Lifetime Version (Spitzer et al, 1978), five patients $(9 \%)$ had psychotic depression and 48 patients had major depression alone. The mean B 12 concentration, measured by radioimmunoassay (Quantphase, Bio-rad, California), for the psychotic group was $181.6 \pm 57.3 \mathrm{pmol} / 1$ (range $107.0 \pm 266.0 \mathrm{pmol} / \mathrm{l})$, while the mean $\mathrm{B} 12$ for the non-psychotic group was $316.7 \pm 105.4 \mathrm{pmol} / \mathrm{l}$ (range $139.0-574.0 \mathrm{pmol} / \mathrm{l}$ ) (normal range $=110$ $630 \mathrm{pmol} / \mathrm{l})$. Using $t$-tests, there was a statistically significant difference between the two groups for B12 levels $(t=2.8$, d.f. $=51, P<0.01)$ but not for folate levels. Furthermore, when several clinical and behavioural variables such as age at onset and duration of depressive illness were entered into a multiple regression with $\mathrm{B} 12$ as the dependent variable, the presence or absence of psychosis contributed significantly to variance in B12 $\left(R^{2}=0.13\right.$, d.f. $\left.=48, P<0.01\right)$.

Patients with psychotic depression may have a lower B12 level than non-psychotic patients. This confirms previous findings that low B12 is associated with mental disturbance (Shovron et al, 1980). This is the first report of which we are aware of a specific association between psychotic depression and lower B12. We have previously shown (Levitt \& Joffe, in preparation) that B12 is not associated with duration of current depression or weight and appetite changes in depression. In addition, B12 depletion may take many months. It is therefore unlikely that nutritional deficit secondary to the anorexia of current depression is primarily responsible for the lower B12. Although low B1 2 may sometimes result from low folate, we did not find a significant difference in folate levels between the psychotic and non-psychotic depressives. Another possible explanation for this difference is that lower B12 predisposes to the development of psychoticsymptoms during a depressiveepisode. This hypothesis needs to be tested on a large population with repeated measures of B12 after recovery.

ANTHONY J. LEVITT

RUSSEL L T. JOFFE

St Michael's Hospital

Toronto, Ontario MSB IW8

Canada
References

Evans, D. L., Edelson, G. A. \& Golden, R. N. (1983) Otganic psychosis without anaemia or spinal cord symptoms in patients with vitamin B12 deficiency. American Journal of Psychiairy, 140, 218-221.

Shovron, S. D., Carney, M. W. P., Chanarin, I. \& Reynolds, E. H. (1980) The neuropsychiatry of megaloblastic anaemia. British Medical Journal, 281, 1036-1038.

Shurman, R. (1967) Vitamin B12 deficiency and psychiatric illness. British Journal of Psychiatry, 113, 252-256.

SPTtzer, R. L., ENDICOTt, J. \& Rorins, E. (1977) Research Diagnostic Criteria for a Selected Group of Functional Disorders (ED 3). New York: New York State Psychiatric Institute.

SPITZER, R. L. \& ENDICOTT, J. (1978) Schectule for Affective Disorders and Schizophrenia - Lifetime Version (SADS-L) (3rd odn). New York: New York State Psychiatric Institute.

\section{Clinical Dementia Rating}

SIR: The Washington University Clinical Dementia Rating (Journal, 1982, 140, 566-572) has been widely adopted. A revision of this staging scale was published in a Letter to the Editor (Journal, 1984, 145, 339).

In order to describe more precisely the rating of questionable dementia (CDR 0.5), our group has recently published a second revision (Mount Sinai Journal of Medicine, 1988, 55, 87-96). Because this change may be of interest to your readers, the newest version is offered here (Table I).

\section{Washington University School of Medicine}

LEONARD BERG

Box 8111

660 South Euclid Avenue

St Louis, MO 63110

USA

\section{Delusional AIDS and Depression}

SIR: In high-risk subjects (drug addicts, homosexuals, anancastic or paranoid personalities) depressive states have been observed in which patients held the delusional belief of having AIDS (Miller et al, 1985; Fleming, 1986). We report a case of a patient showing a delusional idea of death from AIDS.

Case Report: R.M. is a 32-year-old heterosexual male with no previous personal or family history of psychiatric disorder. At the age of $\mathbf{3 0}$ he developed the fear of being affected by AIDS because he occasionally experienced cephalgia, vomiting, and diarrhoea; repeated routine blood tests were always negative. But the patient remained unconvinced, and at the age of 32 he applied for admission to an infectious diseases unit. HIV antibody testing gave negative results. Nevertheless, the patient remained deluded, convinced of his infection, and decided to await death: he therefore stopped work and took to his bed. This behaviour was 